\title{
Early Ambulatory Multidrug Therapy Reduces Hospitalization and Death in High-Risk Patients with SARS-CoV-2 (COVID-19)
}

\author{
Brian Procter ${ }^{1}$, Casey Ross ${ }^{1}$, Vanessa Pickard ${ }^{1}$, Erica Smith ${ }^{1}$, Cortney Hanson ${ }^{1}$, and Peter \\ A. McCullough ${ }^{1}$
}

${ }^{1}$ Affiliation not available

January 7, 2021

\begin{abstract}
Background. There is an emergency need for early ambulatory treatment of COVID-19 in acutely ill patients in an attempt to reduce disease progression and the risks of hospitalization and death. Methods and Results. We recently reported results on 320 high-risk (age > 50 with [?] 1 comorbidity) COVID-19 cases and have updated our results with 549 additional cases in period ending December 16, 2020. Our protocol utilizes at least two agents with antiviral activity against SARS-CoV-2 (zinc, hydroxychloroquine, ivermectin) and one antibiotic (azithromycin, doxycycline, ceftriaxone) along with inhaled budesonide and/or intramuscular dexamethasone. Albuterol nebulizer, inhaled budesonide, intravenous volume expansion with supplemental parenteral thiamine $500 \mathrm{mg}$, magnesium sulfate 4 grams, folic acid 1 gram, vitamin B12 $1 \mathrm{mg}$, are administered for severely ill patients who either present or return to the clinic with severe symptoms. In period 1 (April-September, 2020) 6/320 $(1.9 \%)$ and $1 / 320(0.3 \%)$ patients were hospitalized and died, respectively. In period 2, (September-December, 2020) $14 / 549$ (2.6\%) and 1/549 (0.18\%) were hospitalized and died, respectively. For comparison, we used the Cleveland Clinic COVID-19 hospitalization calculator and based on average age and comorbidities the expected rate of hospitalization for both periods was 18.5\%. The cumulative mortality among confirmed and suspected COVID-19 in Collin, Dallas, Denton, and Tarrant counties was $0.76,1.04,0.90$, and 0.97 . As a result, our early ambulatory treatment regimen was associated with estimated $87.6 \%$ and $74.9 \%$ reductions in hospitalization and death respectively, $\mathrm{p}<0.0001$. Conclusions. We conclude that early ambulatory, multidrug therapy is associated with substantial reductions in hospitalization and death compared to available rates in the community. Prompt ambulatory treatment should be offered to high-risk patients with COVID-19 instead of watchful watching and late-stage hospitalization for salvage therapies.
\end{abstract}

Early Ambulatory Multidrug Therapy Reduces Hospitalization and Death in High-Risk Patients with SARS-CoV-2 (COVID-19)

Brian C. Procter, MD, Casey Ross MSN, APRN, FNP-C, Vanessa Pickard, PA-C, MPAS, Erica Smith, PA-C, MPAS, Cortney Hanson, PA-C, MPAS, Peter A. McCullough, MD, MPH, ${ }^{1} 23$

${ }^{1}$ McKinney Family Medicine, ${ }^{2}$ Baylor University Medical Center, ${ }^{3}$ Baylor Heart and Vascular Institute, ${ }^{4}$ Baylor Jack and Jane Hamilton Heart and Vascular Hospital, Dallas, TX

Address for Correspondence

Peter A. McCullough, MD, MPH

Baylor Heart and Vascular Institute

621 N. Hall St, H030, Dallas, TX USA 75226

peteramccullough@gmail.com

Brian C. Procter, MD, bprocter725@gmail.com 
Casey C. Ross, MSN, APRN, FNP-C, casey.ross1965@gmail.com

Vanessa Pickard, PA-C, MPAS, vanessa.pickard@icloud.com

Erica Smith, PA-C, MPAS, ericarios81@gmail.com

Cortney Hanson, PA-C, MPAS, corthanson@att.net

Peter A. McCullough, MD, MPH, peteramccullough@gmail.com

Word Count: 2462

Funding source(s): none related

Conflict of interest statement for author: nothing to disclose

Authors had access to the data and wrote the manuscript

Running head: Outcomes after Ambulatory Treatment of COVID-19

\begin{abstract}
Background. There is an emergency need for early ambulatory treatment of COVID-19 in acutely ill patients in an attempt to reduce disease progression and the risks of hospitalization and death.

Methods and Results. We recently reported results on 320 high-risk (age $>50$ with [?] 1 comorbidity) COVID-19 cases and have updated our results with 549 additional cases in period ending December 16, 2020. Our protocol utilizes at least two agents with antiviral activity against SARS-CoV-2 (zinc, hydroxychloroquine, ivermectin) and one antibiotic (azithromycin, doxycycline, ceftriaxone) along with inhaled budesonide and/or intramuscular dexamethasone. Albuterol nebulizer, inhaled budesonide, intravenous volume expansion with supplemental parenteral thiamine $500 \mathrm{mg}$, magnesium sulfate 4 grams, folic acid 1 gram, vitamin B12 $1 \mathrm{mg}$, are administered for severely ill patients who either present or return to the clinic with severe symptoms. In period 1 (April-September, 2020) 6/320 (1.9\%) and $1 / 320(0.3 \%)$ patients that were hospitalized and died, respectively. In period 2, (September-December, 2020) 14/549 (2.6\%) and 1/549 $(0.18 \%)$ were hospitalized and died, respectively. For comparison, we used the Cleveland Clinic COVID-19 hospitalization calculator and based on average age and comorbidities the expected rate of hospitalization for both periods was $18.5 \%$. The cumulative mortality among confirmed and suspected COVID-19 in Collin, Dallas, Denton, and Tarrant counties was 0.76, 1.04, 0.90, and 0.97. As a result, our early ambulatory treatment regimen was associated with estimated $87.6 \%$ and $74.9 \%$ reductions in hospitalization and death respectively, $\mathrm{p}<0.0001$.
\end{abstract}

Conclusions. We conclude that early ambulatory, multidrug therapy is associated with substantial reductions in hospitalization and death compared to available rates in the community. Prompt ambulatory treatment should be offered to high-risk patients with COVID-19 instead of watchful watching and late-stage hospitalization for salvage therapies.

Key words: SARS-CoV-2; COVID-19; multidrug; hospitalization; mortality; ambulatory; antiviral; zinc; hydroxychloroquine; ivermectin; doxycycline; azithromycin; vitamin; corticosteroid

The epidemic viral outbreak of SARS-CoV-2 infection (COVID-19) is advancing across the United States unabated as mass vaccination is attempted too late in the pandemic.11McCullough PA, Eidt J, Rangaswami J, Lerma E, Tumlin J, Wheelan K, Katz N, Lepor NE, Vijay K, Soman S, Singh B, McCullough SP, McCullough HB, Palazzuoli A, Ruocco GM, Ronco C. Urgent need for individual mobile phone and institutional reporting of at home, hospitalized, and intensive care unit cases of SARS-CoV-2 (COVID-19) infection. Rev Cardiovasc Med. 2020 Mar 30;21(1):1-7. doi: 10.31083/j.rcm.2020.01.42. PMID: 32259899. There are currently no approved drugs or drug combinations in the U.S. indicated for the ambulatory treatment of COVID-19 or its complications. Unfortunately, there are no potentially conclusive randomized trials of multidrug therapy underway at this time. As with all serious medical conditions, there is a role for empiric treatment in an attempt to reduce fatalities.22McCullough PA, Oskoui R. Early multidrug regimens in new 
potentially fatal medical problems[J]. Reviews in Cardiovascular Medicine, 2020. 21(4): 507-508 This brief report gives an update on real world data and the clinical outcomes associated with an empiric multidrug regimen for confirmed COVID-19 patients who present to a single ambulatory clinic in McKinney, which is located in Colin County, Texas, U.S.

We have previously reported on the methods undertaken by primary care providers consisting of a lead physician (BCP) and four advanced practice practitioners (CR, VP, ES, CH) in their care of acutely ill patients with suspected SARS-CoV-2 infection.33Procter BC, Ross C, Pickard V, Smith E, Hanson C, McCullough PA. Clinical outcomes after early ambulatory multidrug therapy for high-risk SARS-CoV-2 (COVID-19) infection. Reviews in Cardiovascular Medicine, 2020, 21(4): 611-614. In brief, all patients underwent contemporary real-time polymerase chain reaction (PCR) assay tests from anterior nasal swab samples. Risk stratification and advised nutraceuticals were in line with previously published guidance as shown in Figure 1.44McCullough, P. (2020). Innovative Early Sequenced Multidrug Therapy for Sars-Cov-2 (Covid-19) Infection to Reduce Hospitalization and Death. International Journal of Medical Science and Clinical Invention, 7(12), 5139-5150. https://doi.org/10.18535/ijmsci/v7i12.02 All patients received empiric treatment on the first day of presentation in most cases before COVID-19 test results and treatment was continued for those with confirmed COVID-19. Our protocol utilized at least two agents with antiviral activity against SARS-CoV-2 (zinc, hydroxychloroquine, ivermectin) and one antibiotic (azithromycin, doxycycline, ceftriaxone) along with inhaled budesonide and/or intramuscular dexamethasone. Albuterol nebulizer, inhaled budesonide, intravenous volume expansion with supplemental parenteral thiamine 500 $\mathrm{mg}$, magnesium sulfate 4 grams, folic acid 1 gram, vitamin B12 $1 \mathrm{mg}$, were administered for severely ill patients who either present or return to the clinic with severe symptoms.55Flannery AH, Adkins DA, Cook AM. Unpeeling the Evidence for the Banana Bag: Evidence-Based Recommendations for the Management of Alcohol-Associated Vitamin and Electrolyte Deficiencies in the ICU. Crit Care Med. 2016 Aug;44(8):1545-52. doi: 10.1097/CCM.0000000000001659. PMID: 27002274. Additionally, for the severely ill population dexamethasone $8 \mathrm{mg}$ and ceftriaxone 1 gram was administered intramuscularly. All patients had in-person or telemedicine followup at 48 hours and as need depending on the duration and intensity of symptoms.66Colbert GB, Venegas-Vera AV, Lerma EV. Utility of telemedicine in the COVID-19 era. Reviews in Cardiovascular Medicine, 2020, 21(4): 583-587. Hospitalization and death data were collected on followup telemedicine visits or calls to family members.

In period 1 (April-September, 2020) 6/320 (1.9\%) and 1/320 (0.3\%) patients were hospitalized and died, respectively. In period 2, (September-December, 2020) 14/549 (2.6\%) and 1/549 (0.18\%) were hospitalized and died, respectively. For comparison, we used the Cleveland Clinic COVID-19 hospitalization calculator and based on average age and comorbidities the expected rate of hospitalization for both periods was 18.5\%.77Jehi, L., Ji, X., Milinovich, A., Erzurum, S., Merlino, A., Gordon, S., Young, J. B., \& Kattan, M. W. (2020). Development and validation of a model for individualized prediction of hospitalization risk in 4,536 patients with COVID-19. PloS one, 15(8), e0237419. 88https://riskcalc.org/COVID19Hospitalization/ (accessed January 2, 2021) The cumulative mortality among confirmed and suspected COVID-19 in Collin, Dallas, Denton, and Tarrant counties was 0.76, 1.04, 0.90, and 0.97.99https://txdshs.maps.arcgis.com/ (data cutoff December 16, 2020) As a result, our early ambulatory treatment regimen was associated with estimated $87.6 \%$ and $74.9 \%$ reductions in hospitalization and death respectively, $\mathrm{p}<0.0001$.

Our results are consent with those of Zelenko and colleagues who demonstrated that early treatment of COVID-19 in the surge of acute cases in New York City was associated with $84 \%$ and $80 \%$ reductions in hospitalizations and death respectively.1010Derwand R, Scholz M, Zelenko V. COVID-19 outpatients: early risk-stratified treatment with zinc plus low-dose hydroxychloroquine and azithromycin: a retrospective case series study. Int J Antimicrob Agents. 2020 Dec;56(6):106214. doi: 10.1016/j.ijantimicag.2020.106214. Epub 2020 Oct 26. PMID: 33122096; PMCID: PMC7587171. We anticipate that the widespread use of therapeutic antibodies directed against the spike protein of SARS-CoV-2 administered at the site of diagnosis (emergency room, urgent care clinic, nursing home) in addition to the use of anticoagulants in high risk patients will greatly bolster the ambulatory treatment of COVID-19 and have a substantial impact on the rate of hospitalization. ${ }^{4}$ Given the novelty of early ambulatory treatment and the lack of guidelines for 
outpatient treatment of COVID-19, we infer the majority of current hospitalizations and deaths in our region and likely the entire country receive no treatment before hospitalization where salvage treatments are undertaken.1111Hayek SS, Brenner SK, Azam TU, Shadid HR, Anderson E, Berlin H, Pan M, Meloche C, Feroz R, O'Hayer P, Kaakati R, Bitar A, Padalia K, Perry D, Blakely P, Gupta S, Shaefi S, Srivastava A, Charytan DM, Bansal A, Mallappallil M, Melamed ML, Shehata AM, Sunderram J, Mathews KS, Sutherland AK, Nallamothu BK, Leaf DE; STOP-COVID Investigators. In-hospital cardiac arrest in critically ill patients with covid-19: multicenter cohort study. BMJ. 2020 Sep 30;371:m3513. doi: 10.1136/bmj.m3513. PMID: 32998872; PMCID: PMC7525342. Thus, it is important for media and public health messaging to feature early ambulatory treatment as an option for those acutely ill with COVID-19.1212Venegas-Vera AV, Colbert GB, Lerma EV. Positive and negative impact of social media in the COVID-19 era. Reviews in Cardiovascular Medicine, 2020. 21(4): 561-564

In conclusion, our data suggest more than three quarters of hospitalizations and death are avoidable with early ambulatory treatment by primary care teams. Supported by our durable results, we believe early multidrug, ambulatory treatment should be offered as an emergency measure in acutely ill, high-risk COVID19 as a strategy to reduce hospitalization and death. In our opinion, ambulatory treatment is preferable to hospitalization for salvage therapies that are applied too late and associated with complications and in-hospital death.

Figure 1. Early sequential multidrug therapy utilizing risk stratification and available nutraceuticals, appropriately prescribed approved drugs, and U.S. Food and Drug Administration Emergency Use Authorization agents (reproduced with permission from reference) ${ }^{4}$

Figure 2. Comparative results for COVID-19 hospitalizations and death from for early ambulatory treatment of COVID-19 compared with estimated risk of hospitalization and death from the Cleveland Clinic COVID19 Hospitalization Risk Calculator and from the average case fatality rate in the four county surrounding region of the clinic. ${ }^{78} 9$

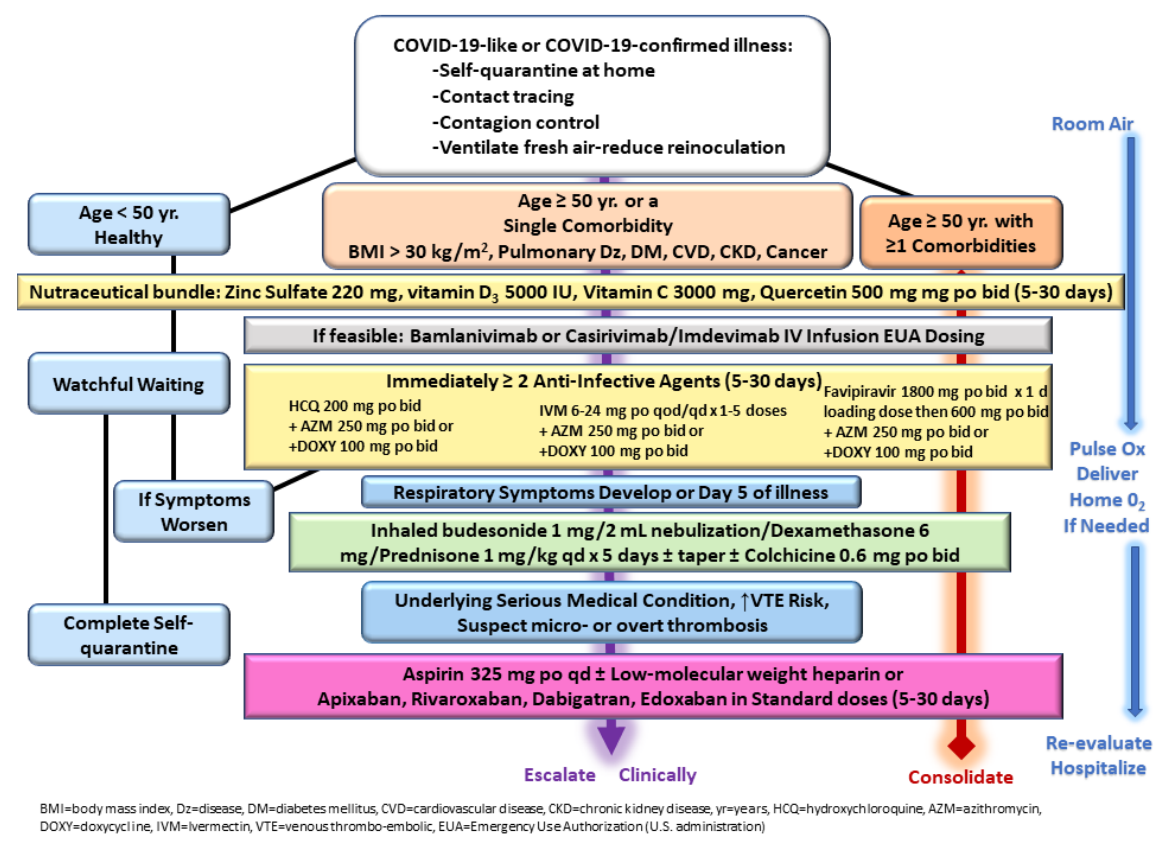




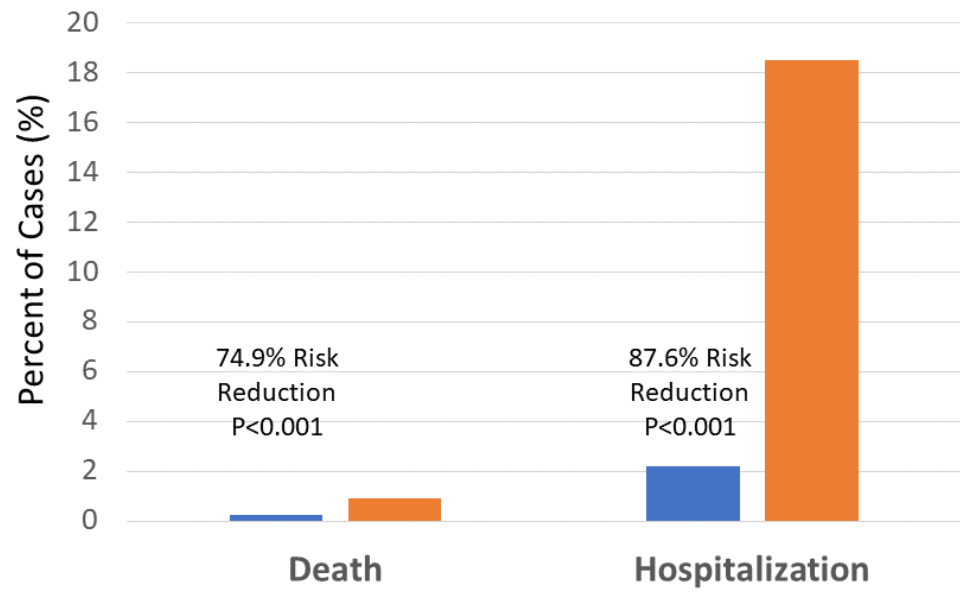

Early Ambulatory COVID-19 Therapy Expected 